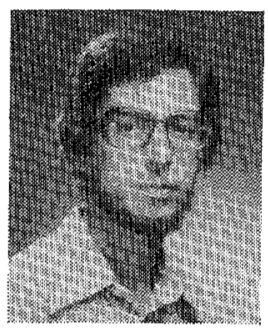

Jean Louis Nicolas was born in Orleans, France, on January 17,1942 . He received the Master's degree in mathematics, agrégation, and Ph.D. degree from the Ecole Normale Supérieure, Paris, France, in 1962,1963, and 1968, respectively.

Between 1964 and 1977 he was successively Assistant at the University of Paris-Sorbonne, Professor at the University of Sherbrooke, Sherbrooke, P.Q., Canada, and Lecturer at the University of Paris-Orsay. Since 1973 he has been Professor at the University of Limoges, Limoges,
France, where he directs a research team on computers in number theory. He has published over 30 research papers on number theory, many concerning optimization problems in integers. He has also published several survey papers on the use of computers in arithmetic, factorization methods, and primality tests. Since 1982 he has been the Dean of the Faculty of Sciences of the University of Limoges.

Dr. Nicolas is a member of the French Mathematics Society (of which he was the Treasurer from 1976 to 1978), of the American Mathematics Society, and of the Association of Former Pupils of the Ecole Normale Supérieure.

\title{
ARMA Model Maximum Entropy Power Spectral Estimation
}

\author{
MIGUEL A. LAGUNAS-HERNÁNDEZ, MEMBER, IEEE, M. EUGENIA SANTAMARÍA-PEREZ, \\ STUDENT MEMBER, IEEE, AND ANÍBAL R. FIGUEIRAS-VIDAL, SENIOR MEMBER, IEEE
}

\begin{abstract}
In this paper we show that the appropriate selection of constraints in the variational formulation of spectral estimation leads to new models and more freedom for the designer. We also illustrate the relationships between the set of constraints and the underlying model in the procedure.

The rest of the paper concentrates on obtaining maximum entropy ARMA models for spectral estimation, using cepstral constraints and correlation constraints simultaneously. The nonlinearity that these kinds of constraints introduce is avoided by a simple linearization, that provides an estimator which is easily implemented. Finally, some examples are given to illustrate the performance of the proposed method.
\end{abstract}

\section{INTRODUCTION}

$\mathrm{T}$ HE variational procedure for spectral analysis consists of minimizing an objective function which depends on the estimator $S(w)$, and that usually has the form $\int F[S(w)] d w$. This optimization is carried out under a set of constraints which are frequently obtained from the periodogram $P(w)=$ $|X(w)|^{2} / N$ of the recorded data in the standard form

$$
\begin{aligned}
& \frac{1}{2 \pi} \int_{-\pi}^{\pi} G[S(w)] \exp (j m w) d w=\frac{1}{2 \pi} \int_{-\pi}^{\pi} G[P(w)] \\
& \quad \cdot \exp (j m w) d w ; \quad|m|<Q .
\end{aligned}
$$

Modifications of the periodogram, like the classical ones proposed in [1], can be used here.

Manuscript received July 5, 1983 ; revised March 12, 1984.

M. A. Lagunas-Hernández and M. E. Santamaría-Perez are with the E.T.S.I. de Telecomunicación, Universidad Politécnica de Barcelona, Barcelona, Spain.

A. R. Figueiras-Vidal is with the E.T.S.I. de Telecomunicación, Universidad Politécnica de Madrid, Madrid 3, Spain.
The constraints that have been employed currently in spectral analysis procedures are those corresponding to $G(\cdot)=\cdot$, i.e., the biased correlation estimate. But we shall see in the sequel that other possibilities can also be of interest.

First, note that the constraints and their number serve to incorporate the specific knowledge we have about the signal under analysis to the final estimator. Through these constraints, we use the information provided by the signal samples ( $N \gg Q$ where $N$ is the register length). This data reduction from $N$ signal data samples to $Q$ (less than $N$ ) constraints is an important drawback in parametric spectral analysis. Under such a reduction, it is decisive to select the type of constraints that reflects best the essential characteristics of the series under analysis.

On the other hand, in consideration of the importance of the constraints and their number, it should be noted that the selection of the objective amounts to deciding how to use this information: a good selection will make adequate use of the information concerning the true spectral density provided by the data samples and represented in the procedure by the constraints. Then, we can say that this pair of choices are closely related: a certain objective will be appropriate for a certain set of constraints, and vice versa. Objective and constraints will determine some characteristics of the resulting estimate like resolution, low sidelobe level, bias in the spectral peaks, underlying spectral model, etc. We can conclude that to select correlation constraints is not necessarily the best procedure in every case, especially if we keep in mind that we need to maintain the number of these constraints below the number of available data samples $(N)$. 
We shall introduce an example to show the versatility we pointed out before and the wide range of possibilities open here. Let us assume that the objective is the entropy [2] $\int \log S(w)$ $d w$ and let us assume three different families of constraints,

$$
\begin{aligned}
& G[P(w)]=P(w) \\
& G[P(w)]=\log P(w) \\
& G[P(w)]=P(w) \cdot \log \{P(w)\}
\end{aligned}
$$

in order to compare the corresponding results.

The basic procedure, in the three cases, is to form the Lagrangian and set its derivative equal to zero. Depending upon the objective function, we obtain the following.

The use of $2 Q+1$ constraints (2a) with $|m|<Q+1$ leads to the well-known AR model for $S(w)$.

$$
S(w)=\left[1 / \sum_{m=-Q}^{Q} b(m) \cdot \exp (-j w m)\right] .
$$

A set of $2 Q$ constraints $(2 \mathrm{~b})(|m| \leqslant Q, m \neq 0)$ and one constraint (2a) $m=0$ will result in an MA model

$$
S(w)=\left[1-\sum_{m=1}^{Q} 2 \cdot b(m) \cdot \cos (m w)\right] / a(0) .
$$

A set of $2 Q+1$ constraints $(2 c)(|m| \leqslant Q)$ will give an AR model for $S(w) \cdot(1+\log S(w))$.

$$
S(w) \cdot(1+\log (S(w)))=\left[\sum_{m=-Q}^{Q} b(m) \cdot \exp (-j w m)\right] .
$$

Clearly, there is not any reason in spectral estimation problems to restrict ourselves to an AR, an MA, or an ARMA model of $S(w)$; the designer can use these models for other function of $S(w)$. Such use of noncorrelation constraints increases the possibilities of parametric and nonparametric spectral analysis [6].

It is obvious that the statistical stability and the degree of nonlinearity introduced in the spectral estimation method have to be considered in selecting the objective and constraints.

In the next section we will explore the alternatives that can be used to implement maximum entropy without forcing AR models.

\section{MAXImum Entropy ARMA Models}

Since we have indicated that cepstral and correlation constraints produce MA and AR models when we maximize the entropy as objective, it would seem that to impose these two families of constraints simultaneously will provide an ARMA model. The reader can see in [3] how the conditions

$$
\begin{array}{cc}
\frac{1}{2 \pi} \int_{-\pi}^{\pi} S(w) \cdot \exp (j m w) \cdot d w=r(m) ; & |m| \leqslant Q \\
\frac{1}{2 \pi} \int_{-\pi}^{\pi} \log [S(w)] \cdot \exp (j n w) \cdot d w=c(n) ; & |n|=1, Q
\end{array}
$$

when $\int \log (S(w)) d w$ is maximized leads to an $\operatorname{ARMA}(Q, Q)$ model, where the cepstrum values contribute to the estimator zeros and the correlation values to the poles. We can represent the ARMA estimator in the form

$$
S(w)=A^{t} \cdot T / B^{t} \cdot T
$$

where

$$
\begin{aligned}
A & =[1, a(1), \cdots, a(Q)]^{t} \\
B & =[b(0), b(1), \cdots, b(Q)]^{t} \\
\boldsymbol{T} & =[1,2 \cdot \cos (w), \cdots, 2 \cdot \cos (Q w)]^{t} .
\end{aligned}
$$

We would like to emphasize two points with respect to this result.

First: as was said in the previous section, maximum entropy can be associated with non-AR models.

Second: we are working with $4 Q+1$ constraints, with which we can introduce more specific information about the signal process than with the $2 Q+1$ correlation constraints.

To find the vectors $\boldsymbol{A}$ and $\boldsymbol{B}$ that satisfy (3a) and (3b) and maximize the entropy is a nonlinear problem that would require nonlinear programming or iterative methods for its solution. These methods would have unknown convergence conditions, and the difficulties would increase if we impose positivity on the resulting spectral estimate $S(w)$. Thus, we will explore here the alternative of linearizing the problem, introducing a computational procedure that needs only FFT's and Levinson's algorithm to obtain the spectral estimate.

To this end, we first impose the autocorrelation constraints. Then, we search for a set of linear equations that corresponds to the cepstral constraints and that provides an approximate (and simple) solution.

\section{ImPOSING CORRElation Constraints}

We obtain the correlation values to be imposed as the $Q+1$ first points of the IDFT of the periodogram. The rest of the autocorrelation sequence is extrapolated by the applied procedure.

Let us assume that the autocorrelation sequence of an ARMA model whose magnitude corresponds to the desired estimator is completely known. Then, we have

$$
A(z) / B(z)=R(z)
$$

where

$$
\begin{aligned}
& A(z)=1+\sum_{q=1}^{Q} a(q) \cdot\left(z^{-q}+z^{q}\right) \\
& B(z)=b(0)+\sum_{q=1}^{Q} b(q) \cdot\left(z^{-q}+z^{q}\right) \\
& R(z)=r(0)+\sum_{q=1}^{\infty} r(q) \cdot\left(z^{-q}+z^{q}\right) .
\end{aligned}
$$

From (6), we can obtain the following relationships among $a(\cdot), b(\cdot)$, and $r(\cdot)$ (see [4]):

$$
1=b(0) \cdot r(0)+2 \cdot \sum_{m=1}^{Q} b(m) \cdot r(m)
$$




$$
\begin{gathered}
a(q)=b(0) \cdot r(q)+\sum_{m=1}^{Q} b(m) \cdot[r(m+q)+r(|m-q|)] ; \\
q=1, Q .
\end{gathered}
$$

(8a) and (8b) can be rewritten in the form denoted by

$$
A=\mathbf{R} \cdot B
$$

where

$$
\mathbf{R}=\mid \begin{array}{cc}
r(0) & 2 r(1) \\
r(1) & r(0)+r(2) \\
\vdots & \vdots \\
r(Q) & r(Q-1)+r(Q+1)
\end{array}
$$

Note that (9) represents $Q+1$ equations with $2 Q+1$ unknowns; thus, we need $Q$ more equations to find these unknowns and these $Q$ equations will be obtained from the cepstral constraints.

On the other hand, the number of autocorrelation values used in (9) is greater than $Q+1$. As already shown, not all of them have to be known, but we do have this information from the periodogram. A different possibility is to use the correlation causal image proposed by Cadzow [5] which formulate the correlation constraints using only $Q+1$ correlation lags. We will select here the first possibility, using the required number of points from the IDFT of the periodogram, although this number will be greater than the number of correlation constraints.

\section{Imposing Cepstral Constraints}

We will try to impose the $Q$ cepstral constraints in an approximate form by means of the following linear equations:

$$
\begin{gathered}
a(q)=b(0) \cdot \hat{r}(q)+\sum_{m=1}^{Q} b(m) \cdot[\hat{r}(m+q)+\hat{r}(|m-q|)] ; \\
q=1, Q
\end{gathered}
$$

where $\hat{r}(\cdot)$ is a pseudocorrelation function to be defined later.

In order to maintain the formulation equivalent to the correlation constraints [see (9)], the set (11) is extended with a new coefficient $\hat{a}(0)$ in such a way that $(12)$ results,

$$
\hat{\boldsymbol{A}}=\hat{\mathbf{R}} \cdot \boldsymbol{B}
$$

where

$$
\hat{A}=[\hat{a}(0), a(1), \cdots, a(Q)]^{t}
$$

and $\hat{\mathbf{R}}$ is formed from $\hat{r}(\cdot)$ as $\mathbf{R}$ was obtained from $r(\cdot)$ in (10).

Matrix $\hat{\mathbf{R}}$ is found in a form similar to that used to compute R from $P(w)$, but starting from a modified periodogram $\widehat{P}(w)$ yet to be selected. In order to make this selection, let us assume that we know the estimate $S(w)$ satisfying all the cepstral constraints; then, we can write

$$
\begin{aligned}
S(w)= & \exp \left[c^{e}(0)+2 \cdot \sum_{q=1}^{Q} c(q) \cdot \cos (q w)\right. \\
& \left.+2 \cdot \sum_{q>Q}^{\infty} c^{e}(q) \cdot \cos (q w)\right]
\end{aligned}
$$

where $\{c(q)\}, q=1, Q$ are the known cepstrum values,

$$
c(q)=\frac{1}{2 \pi} \int_{-\pi}^{\pi}(\log (P(w)) \cdot \exp (j q w) \cdot d w
$$

and

$$
\left.P(w)=\exp \quad c(0)+2 \cdot \sum_{q=1}^{\infty} c(q) \cdot \cos (q w)\right]
$$

with $c^{e}(0)$ and $c^{e}(q),|q|>Q$, the cepstrum values extrapolated by the procedure we use to obtain $S(w)$. It is not difficult to prove [3] that (14) can be rewritten in a form which reveals that cepstrum constraints can be expressed as a datadependent window acting on the periodogram;i.e.,

$$
S(w)=P(w) \cdot \exp [\bar{C}(w)]
$$

where

$$
\bar{C}(w)=\bar{c}(0)+2 \cdot \sum_{q=1}^{\infty} \bar{c}(q) \cdot \cos (q w)
$$

and

$$
\bar{c}(q)=c^{e}(q)-c(q) .
$$

Now, the problem consists of obtaining an estimate $\hat{C}(w)$ of $\bar{C}(w)$, in order to use

$$
\hat{P}(w)=P(w) \cdot \exp [\hat{C}(w)]
$$

for evaluating $\hat{\mathbf{R}}$.

Before doing so, it is worth gaining more insight into the role of cepstrum constraints in parametric spectral estimation. When we relate cepstrum and autocorrelation, we can obtain the following formula:

$$
\frac{d c(m)}{d r(n)}=\frac{1}{2 \pi} \int_{-\pi}^{\pi}\left(\frac{1}{P(w)}\right) \cdot \exp \{j \cdot(m-n) w\} \cdot d w .
$$

This formula is obtained by differentiating (15) with respect to $r(n)$,

$$
\frac{d c(m)}{d r(n)}=\frac{1}{2 \pi} \int_{-\pi}^{\pi}\left[\frac{1}{P(w)}\right] \cdot\left[\frac{d P(w)}{d r(n)}\right] \cdot \exp (j w m) \cdot d w
$$

Then, since $P(w)$ is the discrete Fourier transform of $r(\cdot)$, (21) follows.

Let us underline the following two facts.

1) Since $P(w)$ is nonnegative, (21) reaches its maximum value when $n$ is equal to $m$; i.e., a cepstrum coefficient mainly depends on the corresponding autocorrelation coefficient.

2) Equation (21) confirms the earlier comment relative to 
the contribution of the cepstrum depending essentially on zero locations, since $1 / P(w)$ is the integrand of $(21)$.

Due to these facts, we propose to estimate $c(q)$ in the following manner:

$$
\begin{array}{ll}
\hat{c}(0)=\text { estimate of }\left[c^{e}(0)-c(0)\right]=\alpha \cdot r(0) & \\
\hat{c}(q)=\text { estimate of }\left[c^{e}(q)-c(q)\right]=\alpha \cdot r(q) ; & q=1, N \\
\hat{c}(q)=\text { estimate of }\left[c^{e}(q)-c(q)\right]=0 ; & q>N
\end{array}
$$

where $N$ is the number of data points, and

$$
\alpha=\frac{1}{2 \pi} \int_{-\pi}^{\pi}[1 / P(w)] \cdot d w
$$

a paramter denoting the increment ratio. Then, we can obtain $\hat{C}(w)$ by using (23a)-(23c) with a formula equivalent to (18), and computing $\hat{P}(w)$ according to $(20)$. This procedure allows us to compute matrix $\widehat{\mathbf{R}}$.

\section{Applying the Proposed Method}

Given the sets of equations (9) and (12) for correlation constraints and cepstrum constraints, respectively, we can subtract them to form

$$
(\hat{\mathbf{R}}-\mathbf{R}) \cdot B=E
$$

where

$$
\boldsymbol{E}=[\hat{a}(0)-\mathrm{a}(0), 0, \cdots, 0]^{t} .
$$

Then, computing vector $\boldsymbol{B}$ is equivalent to solving an all-pole problem, but working with $\hat{\mathbf{R}}-\mathbf{R}$. Thus, we can use Levinson's algorithm to obtain $D(z)$, which is related to $B(z)$ by $B(z)=$ $(D(z) \cdot D(1 / z))$. This is possible because $\mathbf{R}-\mathbf{R}$ is a correlation matrix, since $\hat{C}(w)$ is normalized to be greater than zero, in order to guarantee the positiveness of the function $\hat{P}(w)$ $P(w)$. This normalization is reasonable, but there are no obvious reasons to say that $\bar{C}(w)$ has to be positive. Therefore, the above procedure is suggested to allow the use of Levinson's algorithm, forcing the unknown behavior of $\hat{C}(w)$ (normalized to be within 0 and 10 in the examples). From several examples carried out by the authors, it can be concluded that high dynamic ranges for $\hat{C}(w)$ will increase the "cosmetic" resolution of the resulting spectral estimate. This dynamic range is limited by software constraints in the exponentiation of the function $\hat{C}(w)$ involved in computing $\hat{P}(w)$. Simultaneously, the use of Levinson's algorithm ensures a nonnegative result for $B(w)$.

$A(w)$ can be computed by using the procedure proposed by Kay [4]; in this way, we guarantee the nonnegative character of the resulting estimate.

We can summarize the proposed method in the following steps.

1) Compute $r(m)$ from the data as follows:

$$
r(m)=\frac{1}{N} \sum_{n=0}^{N-m} x(n+m) \cdot x(n) .
$$

2) Use the DFT to obtain

$$
\begin{array}{rlrl}
P(l) & =\operatorname{DFT}[r(m)], & l & =0, N-1 \\
\hat{c}(l) / \alpha & =\operatorname{DFT}[\hat{r}(m)], & l=0, N-1
\end{array}
$$

where

$$
\hat{r}(m)= \begin{cases}r(0), & m=0 \\ r(m), & Q+1 \leqslant|m|<N \\ 0, & \text { otherwise. }\end{cases}
$$

3) Compute $\alpha$ and $\hat{P}(l)$ in the form

$$
\sum_{i=0} \mid[1 / P(l)]
$$

and normalize $\hat{C}(l)$ between 0 and 10

$$
\hat{P}(l)=P(l) \cdot \exp [\hat{C}(l)] \text {. }
$$

4) Compute the first $Q+1$ values of

$$
\text { IDFT }[\hat{P}(l)-P(l)] \text {. }
$$

5) Apply the Levinson algorithm to the previous $Q+1$ values to obtain

$$
D(z)=1+\sum_{q=1}^{Q} d(q) \cdot z^{-q}
$$

6) Filter the data record to obtain $A(z)$ according to the procedure reported in [4].

7) Form the spectral estimator as

$$
S(w)=A(w) /\left[D(w) \cdot D^{*}(w)\right] \text {. }
$$

\section{SOME EXAMPLES}

We present here some results from different simulations carried out in order to check the validity of the proposed scheme.

In all examples, we use 256 data signal samples.

The selected experiments are noi exhaustive, of course, since a complete comparison to the currently reported methods for spectral analysis would require an enormous quantity of graphic results. Nevertheless, we have selected significant experiments, to prove the interest of the proposed procedure for spectral analysis.

The considered cases are as follows.

a) AR signal model, with denominator $1-2.76 z^{-1}+$ $3.81 z^{-2}-2.65 z^{-3}+0.92 z^{-4}$. The estimator is $\operatorname{ARMA}(5,5)$.

b) The same plus white noise, $\mathrm{SNR}=5 \mathrm{~dB}$. The estimator is ARMA $(10,10)$.

c) Two coherent sinusoids having normalized frequencies 0.2 and 0.215 with signal-to-noise ratio of $20 \mathrm{~dB}$ each. The estimator is ARMA $(15,15)$.

d) ARMA signal model: $\left(1+0.9 z^{-6}\right) /\left(1-0.9 z^{-6}\right)$. The estimator is ARMA $(10,10)$.

The corresponding results are shown in Figs. 1-4. Note that usually the peaks at the SPD are narrower in the estimate than in the actual spectrum. This effect seems to be associated to the cepstrum constraints in MEM that, even in the case of allpole processes under test, tend to give the poles closer to the unit circle in the estimate than their actual locations. More 


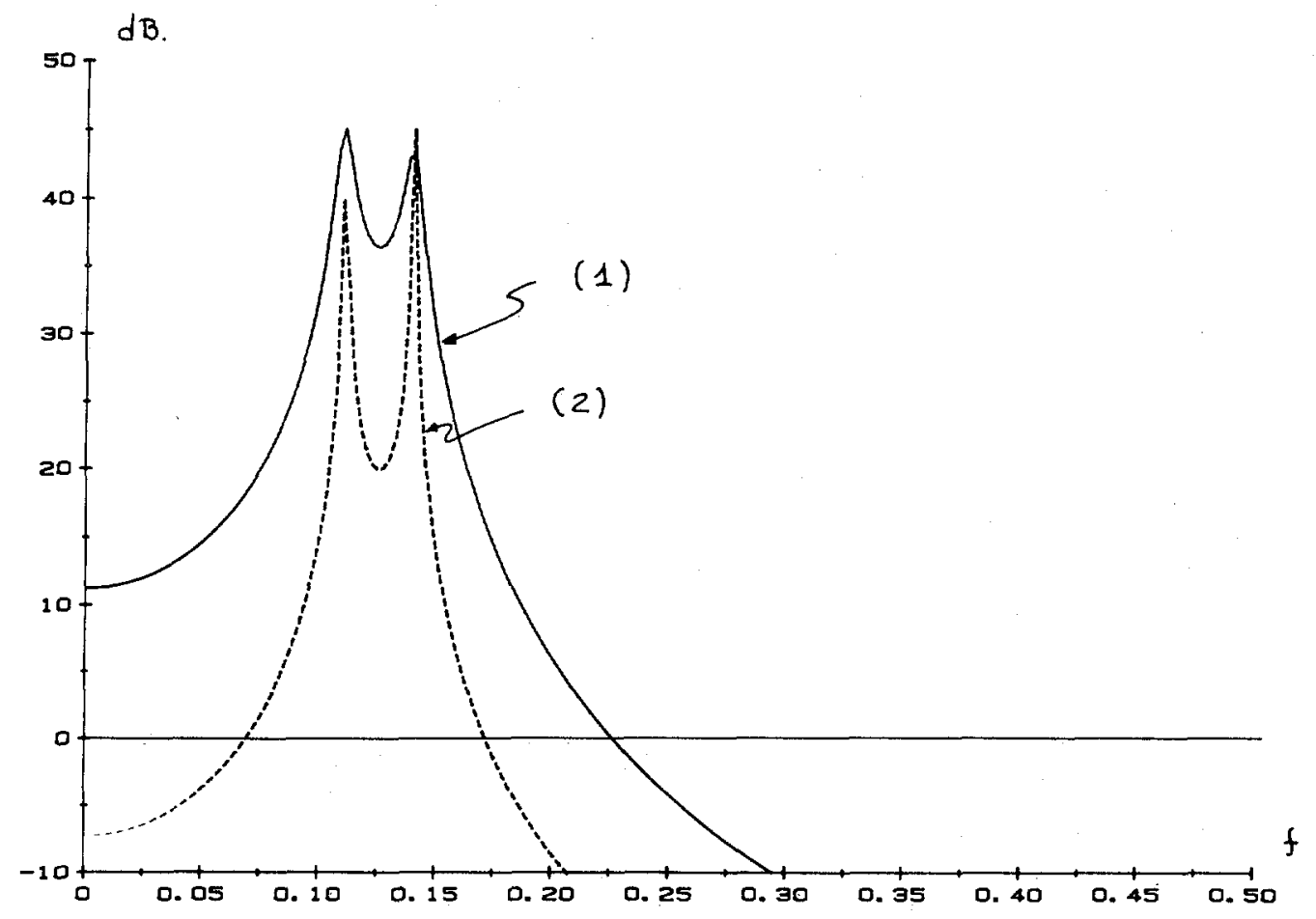

Fig. 1. Case a): 1) actual pure AR(5); 2) ME with correlation and cepstrum constraints ARMA $(5,5)$.

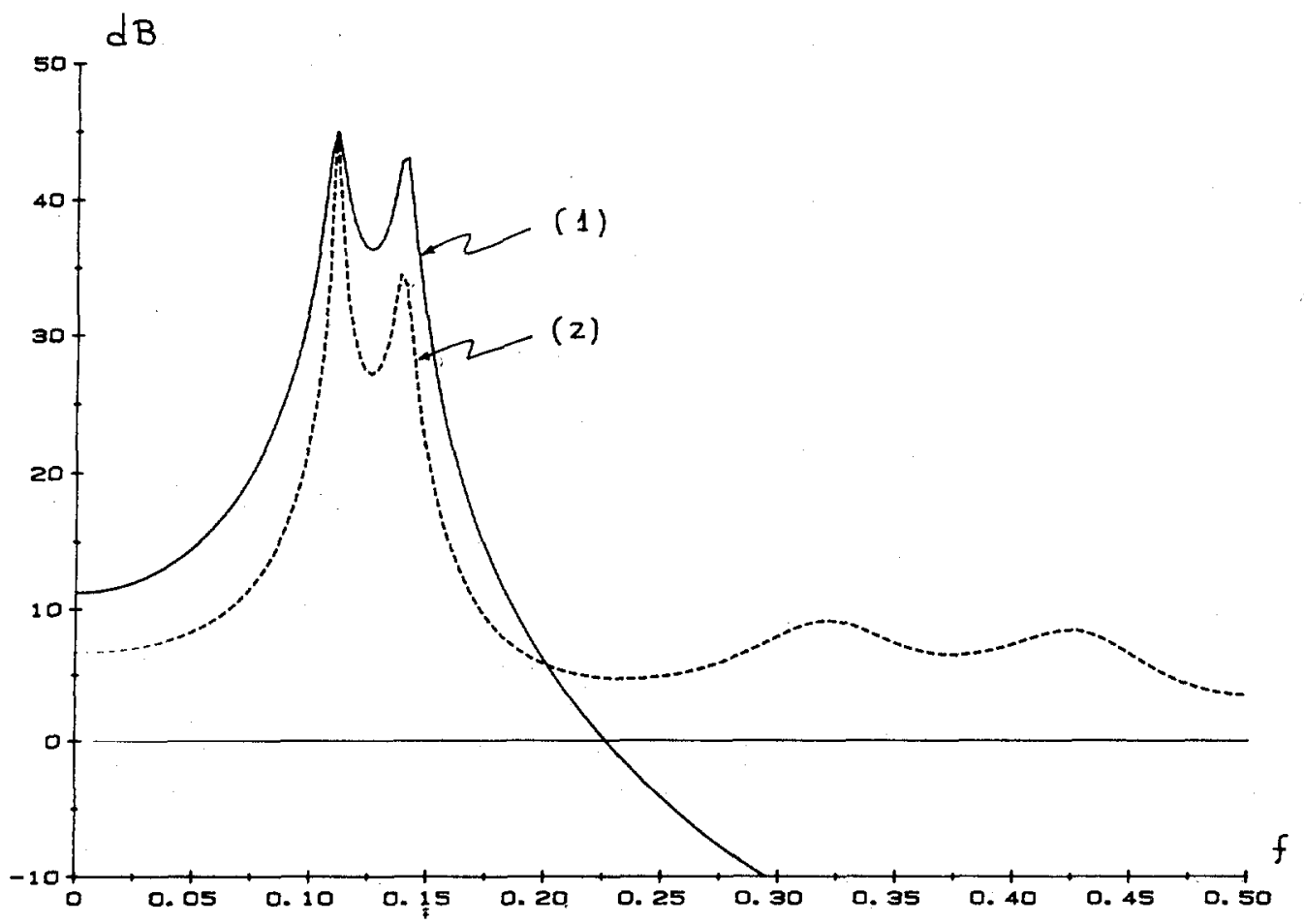

Fig. 2. Case b): 1) actual pure AR(5) without showing the noise level which produces an ARMA (5.5) random process; 2) ME estimate with correlation and cepstrum constraints ARMA $(10,10)$.

specifically, since cepstrum constraints stem from the estimate for $\bar{C}(w)$, the authors realize that the bandwidth of the poles in the resulting estimate decreases as the dynamic range of the normalized $\hat{C}(w)$ increases. Finally, Fig. 5(a) shows the variance of the estimate for 20 trials of signal corresponding to the case of Fig. 2 with SNR equal to $10 \mathrm{~dB}$, and the average (Fig. 5(b), dashed line) compared to the actual AR spectrum (continuous line) without noise. It is worthwhile to note that this plot reflects the compromise between variance and the resolution that the procedure exhibits in all the examples; other 


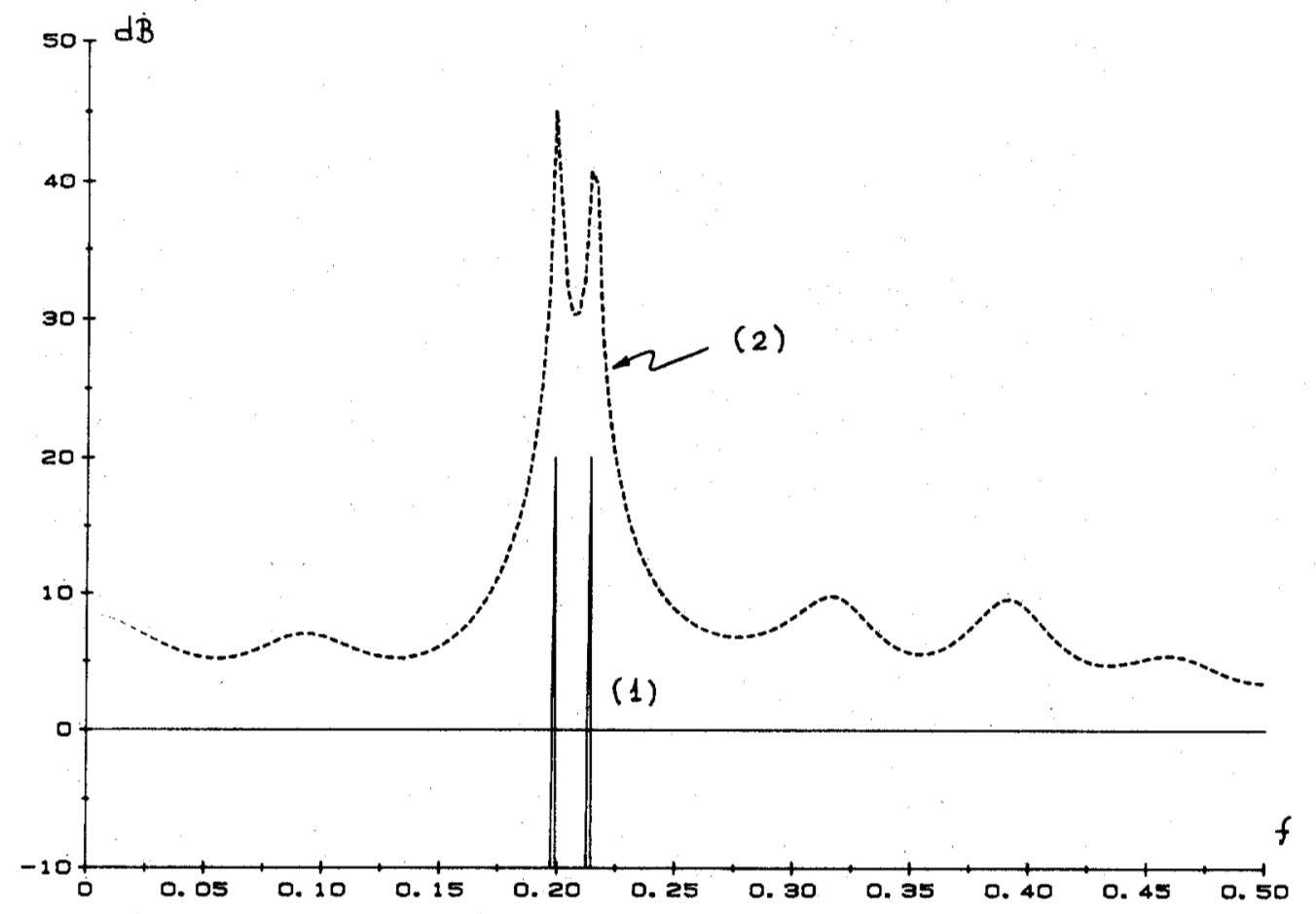

Fig. 3. Case c): 1) actual pure tones at $20 \mathrm{~dB}$ signal-to-noise ratio; 2) ME estimate with autocorrelation and cepstrum constraints ARMA $(15,15)$.

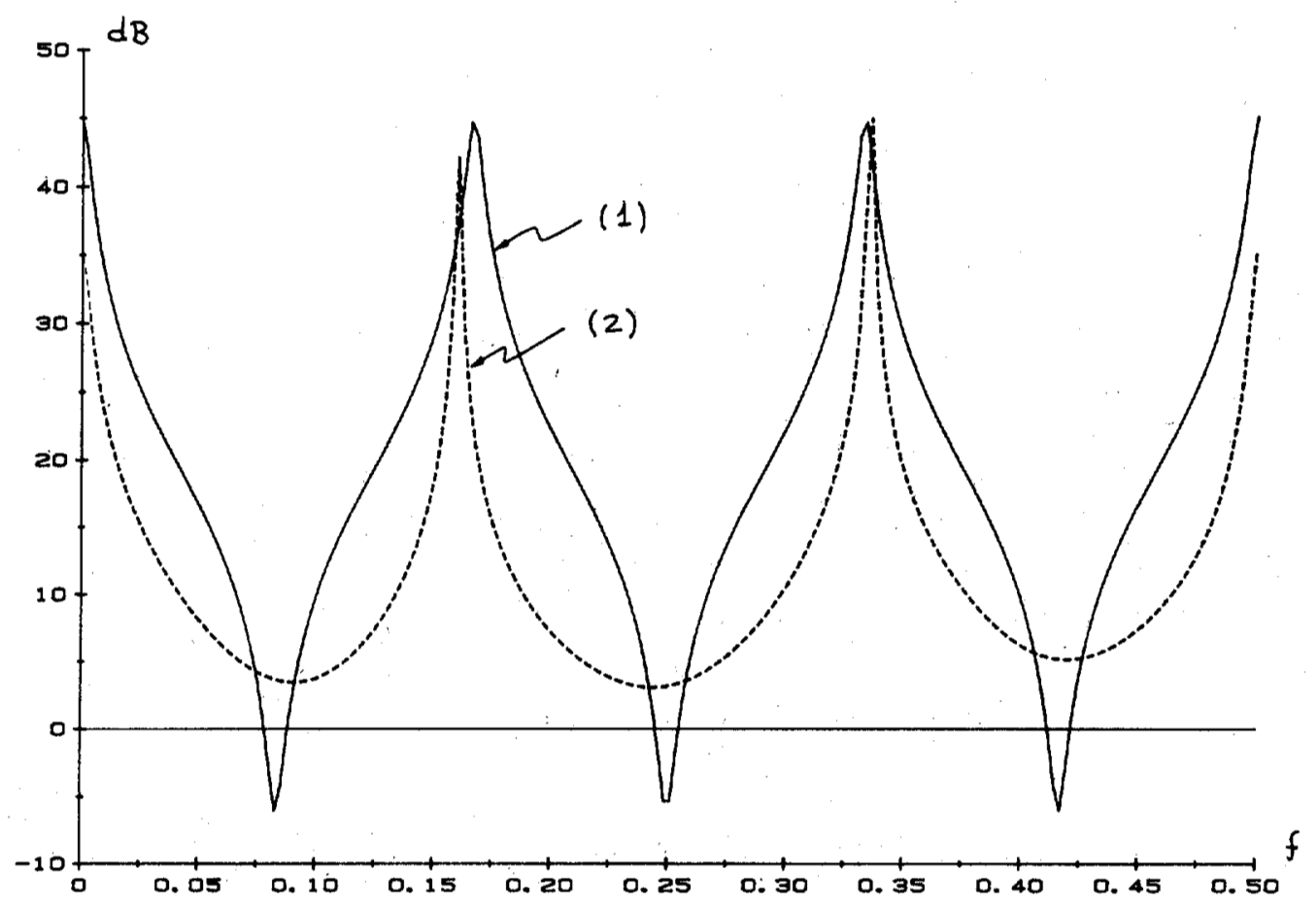

Fig. 4. Case d): 1) actual ARMA $(6,6) ; 2)$ ME estimate with autocorrelation and cepstrum constraints ARMA $(10,10)$.

compromises could be obtained by changing the dynamic range for the estimate of $C(w)$.

\section{CONCLUSIONS}

We have presented a discussion about the use of the objective function and constraints in variational approaches to spectral analysis that opens new possibilities and generalizes previous methods.

Starting from this discussion, and following the lines of previous works, we have considered an ARMA model that maximizes the entropy under simultaneous correlation and cepstrum constraints by introducing some reasonable approxi- 


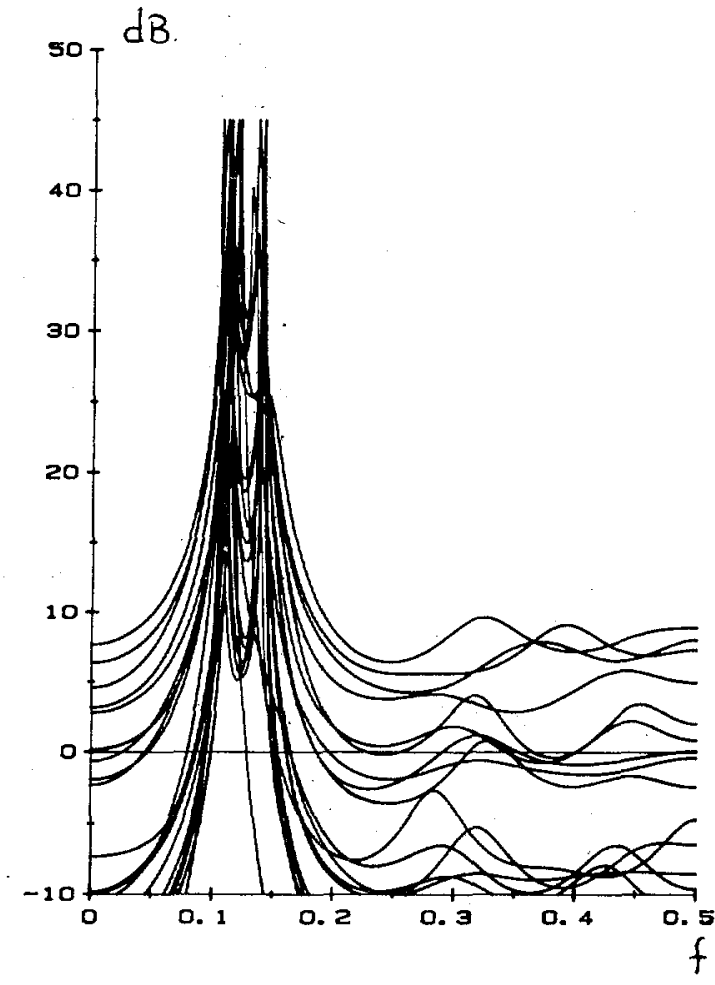

(a)

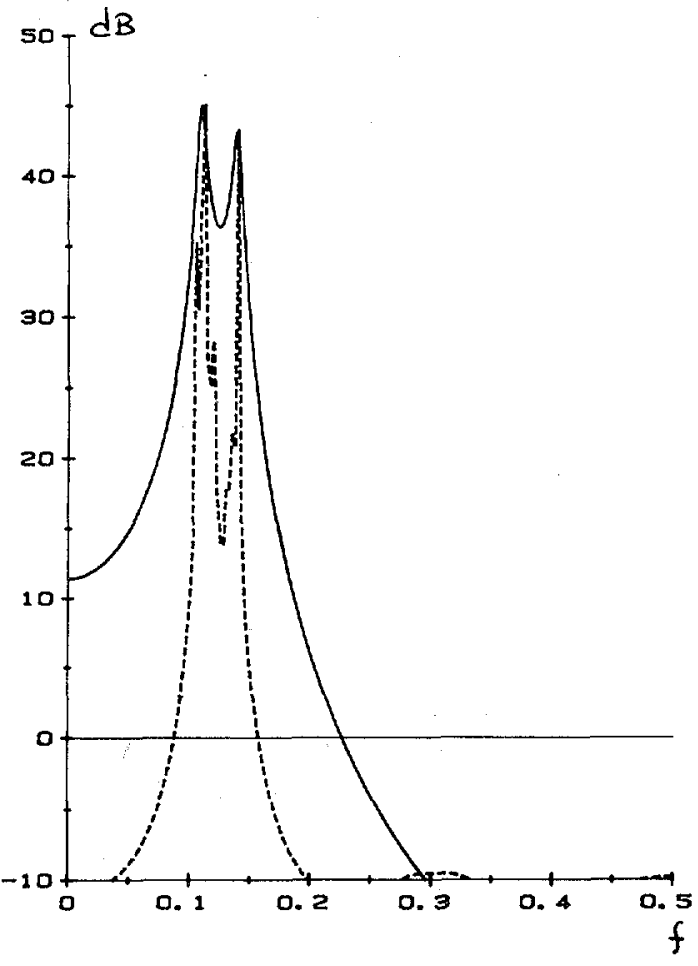

(b)

Fig. 5. (a) Same example as Fig. 2, SNR equal to $20 \mathrm{~dB}$, with 20 trials of data records 256 samples each. (b) Averaged (- $\rightarrow$ ) and actual AR spectrum without noise $(-)$.

mation. The use of cepstrum constraints in order to locate zeros of the model is emphasized. Finally, some examples serve to show the performance of this particular method.

\section{REFERENCES}

[1] P. D. Welch, "The use of fast Fourier transform for the estimation of power spectra: A method based on time averaging over short, modified periodograms," IEEE Trans. Audio Electroacoust., vol. AU-15, pp. 70-73, June 1967.

[2] J. P. Burg, "Maximum entropy spectral analysis," Ph.D. dissertation, Dep. Geophys., Stanford Univ., Stanford, CA, 1975.

[3] M. A. Lagunas, "Cepstrum constraints in ME spectral estimation," in Proc. Int. Conf. Acoust., Speech, Signal Processing, Boston, MA, Apr. 1983 , pp. 1442-1445.

[4] S. Kay, "A new ARMA spectral estimator," IEEE Trans. Acoust., Speech, Signal Processing, vol. ASSP-28, pp. 585-588, Oct. 1980.

[5] J. A. Cadzow, "High performance spectral estimator: A new ARMA method," IEEE Trans. Acoust., Speech, Signal Processing, vol. ASSP-28, pp. 524-529, Oct. 1980.

[6] C. Nadeu and M. Bertran, "Two methods of spectral estimation with rational log spectrum," in Proc. EURASIP-83, Erlangen, West Germany, Sept. 1983, paper D2.5.

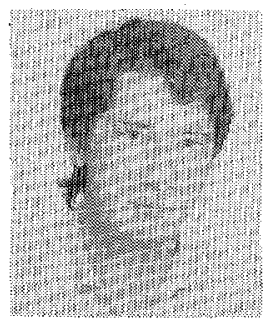

Miguel A. Lagunas-Hernández (S'73-M'78) was born in Madrid, Spain, in October 1951. He received the Telecommunication Engineer Degree from the E.T.S.I. de Telecomunicación, Universidad Politécnica de Madrid, Madrid, in 1973, and the Doctorate in telecommunications engineering from the Universidad Politécnica de Barcelona, Barcelona, Spain, in 1976.

After his graduation he became Professor of Signal Processing in Communications at the E.T.S.I. de Telecomunicación, Barcelona, teach- ing courses in electronics, network analysis and synthesis, communications, and applications of signal processing. During 1981-1982 he was on leave with a Fullbright grant at the Department of Electrical Engineering, University of Colorado, Boulder. His research activities have been centered on signal processing, especially on spectral analysis and adaptive systems.

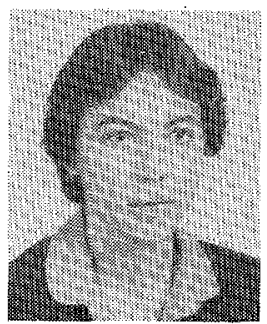

M. Eugenia Santamaría-Perez ( $S^{\prime} 83$ ) was born in Burgos, Spain, on October 1,1960. She received the Telecommunication Engineer degree from the E.T.S.I. de Telecomunicación, Universidad Politécnica de Barcelona, Barcelona, Spain, in 1983.

She is pursuing the Doctorate in telecommunication engineering at the Universidad Politécnica de Barcelona. Currently she is a full-time Research Assistant. Her current research activities are in spectral and cross-spectral estimation.

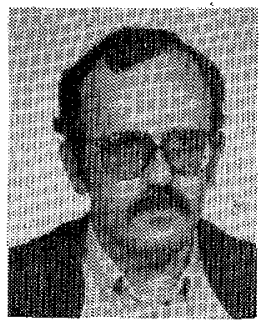

Aníbal R. Figueiras-Vidal (S'74-M'76-SM'84) was born in Vigo, Spain, on May 6, 1950. He received the Telecommunication Engineer degree from the E.T.S.I. de Telecomunicación, Polytechnic University of Madrid, Madrid, Spain, and the Telecommunication Doctor degree from the E.T.S.I. de Telecomunicación, Polytechnic University of Barcelona, Barcelona, Spain. He received the National Award to Graduation, and an Award for his Doctorate.

He is now a Professor at the E.T.S.I.T. Madrid, where he occupies a Vice-fead position. His current research fields ot interest are digital signal processing methods and applications, and communications theory and systems. 